\title{
The recruitment of the U5 snRNP to nascent transcripts requires internal loop 1 of U5 snRNA
}

\author{
Rebecca Kim • Joshua Paschedag • \\ Natalya Novikova $\cdot$ Michel Bellini
}

Published online: 22 November 2012

(C) Springer Science+Business Media Dordrecht 2012

\begin{abstract}
In this study, we take advantage of the high spatial resolution offered by the nucleus and lampbrush chromosomes of the amphibian oocyte to investigate the mechanisms that regulate the intranuclear trafficking of the U5 snRNP and its recruitment to nascent transcripts. We monitor the fate of newly assembled fluorescent U5 snRNP in Xenopus oocytes depleted of U4 and/or U6 snRNAs and demonstrate that the U4/U6.U5 tri-snRNP is not required for the association of U5 snRNP with Cajal bodies, splicing speckles, and nascent transcripts. In addition, using a mutational analysis, we show that a non-functional U5 snRNP can associate with nascent transcripts, and we further characterize internal loop structure 1 of U5 snRNA as a critical element for licensing U5 snRNP to target both nascent transcripts and splicing speckles. Collectively, our data support the model where the recruitment of snRNPs onto pre-mRNAs is independent of spliceosome assembly and suggest that U5 snRNP may promote the association of the U4/ U6.U5 tri-snRNP with nascent transcripts.
\end{abstract}

Responsible Editor: Herbert Macgregor.

R. Kim $\cdot$ J. Paschedag $\cdot$ N. Novikova $\cdot$ M. Bellini $(\bowtie)$ Department of Cell and Developmental Biology, University of Illinois at Champaign-Urbana, 601 S Goodwin Avenue, Room B107 CLSL, Urbana, IL 61801, USA

e-mail: bellini@illinois.edu
Keywords Cajal bodies · Lampbrush chromosomes . RNA splicing $\cdot$ spliceosome $\cdot$ snRNAs

$\begin{array}{ll}\text { Abbreviations } \\ \text { CB } & \text { Cajal body } \\ \text { DAPI } & \text { 4',6-Diamidino-2-phenylindole } \\ \text { Fmol } & \text { Femtomole } \\ \text { IGC } & \text { Interchromatin granule cluster } \\ \text { IgG } & \text { Immunoglobulin G } \\ \text { IL } & \text { Internal loop } \\ \text { LBC } & \text { Lampbrush chromosomes } \\ \text { PBS } & \text { Phosphate-buffered saline } \\ \text { PCR } & \text { Polymerase chain reaction } \\ \text { RNAPII } & \text { RNA polymerase II } \\ \text { RNP } & \text { Ribonucleoprotein } \\ \text { SL } & \text { Stem loop } \\ \text { SnRNP } & \text { Small nuclear ribonucleoprotein particle } \\ \text { snRNA } & \text { Small nuclear RNA } \\ \text { WT } & \text { Wild type }\end{array}$

\section{Introduction}

One of the most prominent features of the lampbrush chromosomes (LBCs) is their very high level of RNA polymerase II (RNAPII) activity (reviewed in Callan 1986; Morgan 2002; see Macgregor ibid). This property is structurally showcased by numerous transcription units in the form of loops that are projected laterally, away from the relatively condensed 
chromosomal axes. While individual loops are easily distinguished using a light microscope, it is important to note that their chromatin axes are only revealed by labeling with antibodies against DNA (see Morgan, ibid) or proteins associated with DNA, such as histones (Austin et al. 2009) and active RNAPII (Gall et al. 1999, see Gall ibid). In essence, the lateral loops correspond to nascent RNAPII transcripts that are associated with numerous RNA processing and export factors. Together, they create a ribonucleoprotein matrix, which is dense enough to be seen by phase contrast or DIC, surrounding a highly decondensed chromatin axis. LBC loops, therefore, represent a unique cellular system to investigate the mechanisms controlling the major steps in RNA transcription and processing, particularly the recruitment of the various machineries involved in these processes. Indeed, it has become well accepted that pre-mRNA transcription and maturation, including splicing, are two interrelated processes in all eukaryotes (Bentley 2005; de Almeida and Carmo-Fonseca 2008; Martins et al. 2011).

A critical step in pre-mRNA maturation is the removal of introns and ligation of exons by the spliceosome, a multi-subunit and dynamic enzyme (Zhou et al. 2002; Jurica and Moore 2003; Nilsen 2003; Patel and Steitz 2003). At the heart of the spliceosome are its catalytic components, the five major small nuclear ribonucleoproteins U1, U2, U4, U5, and U6 snRNPs. Each spliceosomal snRNP consists of a small nuclear RNA (snRNA) associated with a specific set of proteins. The biogenesis of most snRNPs follows a complex maturation pathway that involves both a cytoplasmic and a nuclear phase (Patel and Bellini 2008). Within the nucleus, snRNPs are often detected in association with several discrete domains such as Cajal bodies (CBs), nucleoli, and interchromatin granule clusters (IGCs). While the functional implications of these associations are not immediately clear, the current view is that these domains are involved in the maturation and/or storage of snRNPs and other processing factors prior to their recruitment to nascent transcripts. Previously, we demonstrated that the recruitment of the splicing snRNP to nascent transcripts is independent of the spliceosomal assembly. Additionally, we showed that discrete elements of the $\mathrm{U} 1$ and $\mathrm{U} 2$ snRNPs control their intranuclear trafficking and recruitment to nascent transcripts (Patel et al. 2007; Paschedag et al., in revision). Here, we extend our analysis to the U5 snRNP, which integrates into the spliceosome as part of the U4/U6-U5 tri-snRNP complex. Interestingly, we find that the U5 snRNP still targets nascent transcripts in the absence of the U4 and/or U6 snRNPs. In addition, we define the small internal loop 1 (IL1) of U5 snRNA as a critical element for the association of the U5 snRNP with both IGCs and nascent transcripts.

\section{Materials and methods}

In vitro transcription and labeling

The cDNA clone coding for the full-length Xenopus laevis U5 snRNA was generously provided by Dr. Joseph Gall and was used to generate all templates for in vitro transcription of the wild-type and mutant U5 snRNAs. DNA templates were obtained by PCR, and in all cases, the $\mathrm{T} 3$ promoter was introduced immediately upstream of the sequence to be transcribed. DNA primers (Integrated DNA Technologies) used were as follows (T3 promoter is underlined):

U5 WT: 5'CGGAATTCAATTAACCCTCAC TAAAGGG and 3'ATACCTGGTGTGA ACCAGGCTTC

U5 $\Delta 5^{\prime}$ 47: 5'CGAATTAACCCTCACTAAAG GA A A G A T T C CGTGG and 3'ATACC TGGTGTGAACCAGGCTTC

U5 $\triangle \mathbf{5}^{\prime}$ '68: CGAATTAACCCTCACTAAAGGC GACCATGAGTTTCG and 3'ATACCTGGT GTGAACCAGGCTTC

U5 $\triangle 3^{\prime}$ '96: 5'CGGAATTCAATTAACCCTCAC TAAAGGG and 3'TTCAAAAAATTGAACGA AACTCATGGTCG

DSL1: Step 1: 5'CCTCTGGTTTCTCTTCA A A T T C G A A T A A A T C T T T T T C G A A A GATTTCCG and 3'ATACCTGGTGTGAA CCAGGCTTC;

Step 2: 5'AATTAACCCTCACTAAAGGA TACTCTGGTTTCTCTTCAAATTCG and 3'-A TACCTGGTGTGAACCAGGCTTC

DIL1: Step 1: 5'AATTAACCCTCACTAAA GGATACTTTTCTCTTCAAATTCGAAT and 3' $\bar{T}$ G A A C C A G G C T T C A A A A A A T T GAACGAAACTTTCCTCTCCACGG

S tep 2: 5' A AT TAA C C C T CACTAAA G GATACTTTTCTCTTCAAATTCGAAT and 3'A TACCTGGTGTGAACCAGGCTTC 
$\Delta$ IL2: Step 1: 5'CGCCTTTTACTAAAGATGA GAGGAACGACCAT and 3'ATACCTGGT GTGAACCAGGCTTC;

Step 2: 5'AATTAACCCTCACTAAAGG ATACTCTGGTTTCTCTTATCTTTCGCCTTT TAC and 3'ATACCTGGTGTGAACCAGGCTTC

Amplified DNA templates were gel-purified using $0.45 \mathrm{~mm}$ cellulose acetate spin-X filters (Corning Inc.), phenol extracted, and ethanol precipitated before transcription. Fluorescently labeled U5 snRNA and mutants were synthesized using T3 polymerase (Stratagene) in the presence of $25 \mu \mathrm{M}$ fluorescein12-UTP (Roche), $625 \mu \mathrm{M}$ ATP and CTP, $312.5 \mu \mathrm{M}$ UTP, $250 \mu \mathrm{M}$ GTP, and $1.25 \mathrm{mM} \mathrm{m} 7 \mathrm{G}\left(5^{\prime}\right) \mathrm{ppp}\left(5^{\prime}\right) \mathrm{G}$ cap analog (New England Biolabs, Inc.). Reactions were carried out for $3 \mathrm{~h}$ at $37^{\circ} \mathrm{C}$, treated with RQ1 RNase-free DNase (Fisher Scientific) for $10 \mathrm{~min}$ at $37^{\circ} \mathrm{C}$, and newly made transcripts were purified on NucAway Spin columns (Ambion).

\section{Oocytes and microinjections}

Fragments of ovary were surgically removed from female adult frogs that were anesthetized in $0.15 \%$ tricaine methane sulfonate (MS222; Sigma-Aldrich). Oocytes were defolliculated for $2 \mathrm{~h}$ at room temperature in saline buffer OR2 (Wallace et al. 1973) containing $0.2 \%$ collagenase (type II; Sigma-Aldrich). OR2 buffer is $82.5 \mathrm{mM} \mathrm{NaCl}, 2.5 \mathrm{mM} \mathrm{KCl}, 1.0 \mathrm{mM}$ $\mathrm{CaCl}_{2}, 1.0 \mathrm{mM} \mathrm{MgCl} 2,1.0 \mathrm{mM} \mathrm{Na} \mathrm{HPO}_{4}$, and $5.0 \mathrm{mM}$ HEPES. Stage IV-V oocytes were subsequently isolated and maintained at $18{ }^{\circ} \mathrm{C}$ in OR2. All injections were performed directly into the cytoplasm of oocytes using 10-20 fmol of RNA, with volumes no greater than $30 \mathrm{~nL}$. For the $\mathrm{U} 4$ and $\mathrm{U} 6$ depletion experiments, $50 \mathrm{ng}$ of U4d and U6f oligonucleotides (Integrated DNA Technologies) was injected per oocyte $4 \mathrm{~h}$ prior to the injection of the fluorescent U5 WT snRNA. The U4d sequence, TATTGG GAAAAGTTT, is complementary to nucleotides 66-80 of U4 snRNA. The U6f sequence, TCG TTCCAATTTTAG, is complementary to nucleotides 25-39 of U6 snRNA. Glass needles were prepared using a horizontal pipette puller (P-97; Sutter Instruments Co.). Injections were performed under a dissecting microscope (Leica) using the nanojetII micro-injector from Drummond.
Nuclear spreads and immunofluorescence

Nuclear spreads were prepared as described in (Patel et al. 2007). Preparations were fixed in phosphatebuffered saline (PBS) $(137 \mathrm{mM} \mathrm{NaCl}, 2.7 \mathrm{mM} \mathrm{KCl}$, $8 \mathrm{mM} \mathrm{Na}_{2} \mathrm{HPO}_{4}, 1.46 \mathrm{mM} \mathrm{KH}_{2} \mathrm{PO}_{4}, \mathrm{pH}$ 7.2) containing $2 \%$ paraformaldehyde and $1 \mathrm{mM} \mathrm{MgCl}_{2}$, for $1 \mathrm{~h}$ at room temperature, and blocked in PBS with $0.5 \%$ BSA (Sigma-Aldrich) $+0.5 \%$ gelatin (from cold water fish) for $10 \mathrm{~min}$. The fluorescent signal emitted by fluorescein-labeled RNAs was amplified as follows: Spread preparations were incubated with primary antibody Alexa Fluor 488 anti-fluorescein (Invitrogen) diluted at $2.5 \mu \mathrm{g} / \mathrm{ml}$, washed with PBS, and then incubated with secondary antibody Alexa Fluor 488 goat anti-rabbit IgG (Invitrogen) at $2.5 \mu \mathrm{g} / \mathrm{ml}$. Spreads were washed again with PBS and mounted in $50 \%$ glycerol in PBS $+1 \mathrm{mg} / \mathrm{ml}$ phenylenediamine and $10 \mathrm{pg} / \mathrm{ml}$ DAPI. Microscopy was performed using a fluorescent microscope (DMR; Leica) and a Fluotar $100 \times$ NA 1.30 oil objective (Leica). Images were captured with a Retiga EXI monochrome CCD camera (QImaging) and In Vivo software (version 3.2.0, Media Cybernetics), processed with Photoshop CS version 8.0 (Adobe), and assembled with InDesign CS version 3.0 (Adobe).

Northern blotting

Two sets of ten oocytes were injected with the U4d and U6f oligonucleotides, and ten uninjected oocytes were used as a control. After $4 \mathrm{~h}$ of incubation at $18{ }^{\circ} \mathrm{C}$, the nuclei were collected and homogenized in $10 \mathrm{mM}$ Tri-HCl, pH 8.0, 1 mM EDTA, and $0.2 \%$ sodium dodecyl sulfate. Total RNAs were phenol-extracted and ethanol-precipitated. Northern blots were performed as described in Patel et al. (2007) with following modifications to the protocol: The membranes were crosslinked at $120 \mathrm{~mJ} / \mathrm{cm}^{2}$, and antisense U4, U5, and U6 snRNAs were used as probes.

\section{Results}

Newly assembled U5 snRNPs associate with CBS, $I G C s$, and $L B C$ loops In a recent work, we showed that injection of fluorescent U2 snRNA into the cytoplasm of Xenopus oocytes results in the formation of two populations of nuclear U2 snRNPs. Fully functional 
U2 snRNPs target IGCs and nascent transcripts. In contrast, incompletely assembled U2 snRNPs accumulate in CBs (Paschedag et al., in revision). In addition, experimental evidence strongly supporting a similar model for the U1 snRNP already exists (Patel et al. 2007). Here, we monitored the fate of newly assembled U5 snRNPs. Capped, fluorescently labeled U5 snRNAs were synthesized in vitro and injected into the cytoplasm of stage IV-V Xenopus oocytes. The subnuclear distribution of newly assembled U5 snRNPs was then monitored over time using immunofluorescence microscopy on nuclear spreads. CBs were the only structures labeled after $1 \mathrm{~h}$ of incubation, and over time a weaker but specific signal could be detected on nucleoli, IGCs, and the LBC loops. After $18 \mathrm{~h}$ of incubation, these structures were readily detectable (although nucleoli consistently displayed the weakest signal) and CBs displayed the highest concentration of U5 snRNPs (Fig. 1; magnified views).

The recruitment of U5 SnRNP to nascent transcripts does not require U4 or U6 snRNPs In contrast to the U1 and U2 snRNPs that are recruited to nascent transcripts as individual particles, U5 snRNP is known to further assemble into a U4/U6-U5 tri-snRNP complex prior to its recruitment for spliceosomal assembly. To test how the formation of the tri-snRNP influences the association of U5 snRNPs with CBs, IGCs, and nascent transcripts, we injected fluorescent U5 snRNA into oocytes depleted of U4 and/or U6 snRNAs. Two distinct anti-sense DNA oligonucleotides, U4d and U6f, were used to target the RNase H-mediated degradation of U4 and U6 snRNAs, respectively. Northern blots were performed to monitor the depletion of either U4 or U6 (Fig. 2). Surprisingly, we found that the absence of the U4/U6 di-snRNP does not affect the sub-nuclear distribution of newly assembled U5 snRNPs (Fig. 2), demonstrating that the formation of the U4/U6.U5 trisnRNP is not required for the recruitment of U5 snRNP to nascent transcripts. In addition, since the U4/U6.U5 tri-snRNP is required for spliceosomal activity, this result agrees with our previous work demonstrating that the splicing activity present on the LBC loops does not direct the recruitment of snRNPs, including U5 snRNPs, to nascent transcripts (Patel et al. 2007).

Distinct elements of the U5 snRNP regulate its subnuclear distribution These data suggested that discrete element
Fig. 1 Newly assembled U5 snRNPs associate with CBs, IGCs, and LBC loops. Fluorescently labeled U5 snRNAs were injected into the cytoplasm of oocytes, and nuclear spreads were prepared 18 h later. Newly formed snRNPs were rapidly targeted to IGCs (asterisks), nascent transcripts, and CBs (arrows) where they accumulated at a very high concentration (magnified views). A weaker, but specific signal was also observed within nucleoli (arrow heads)
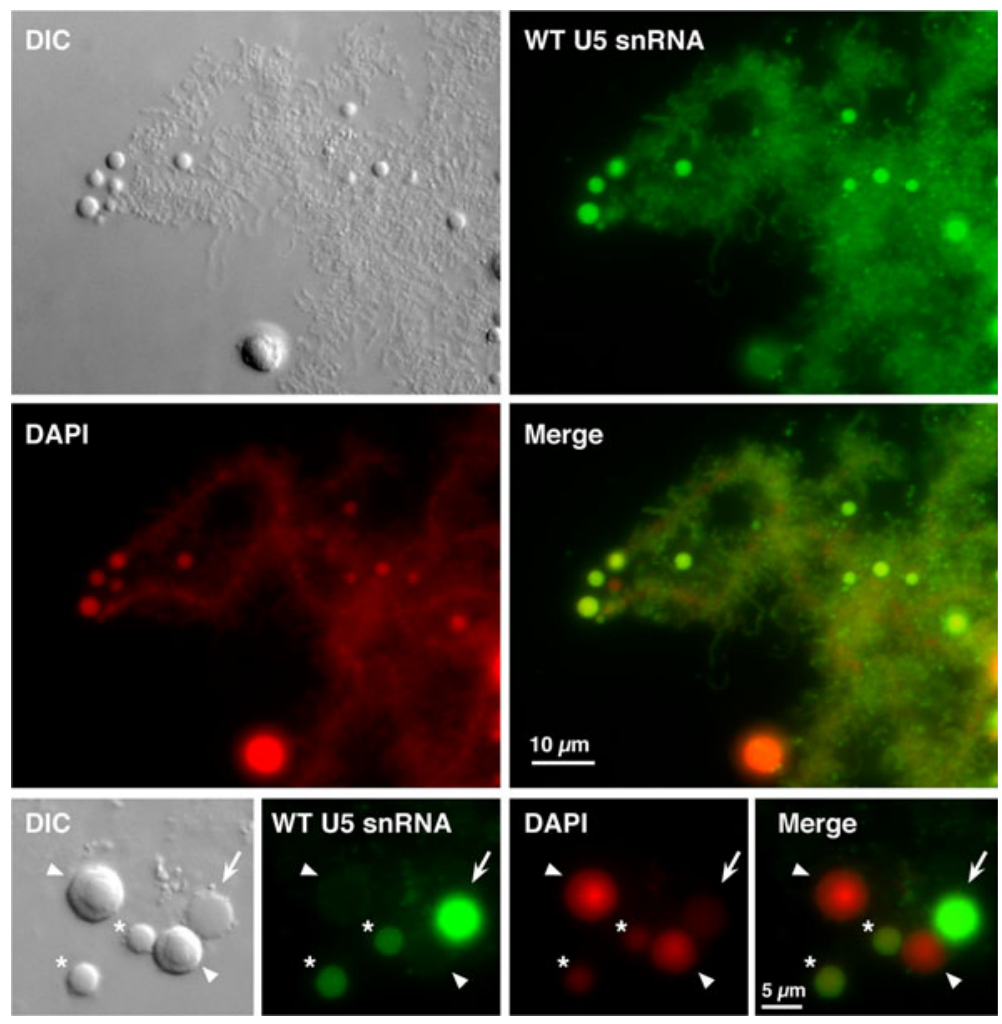

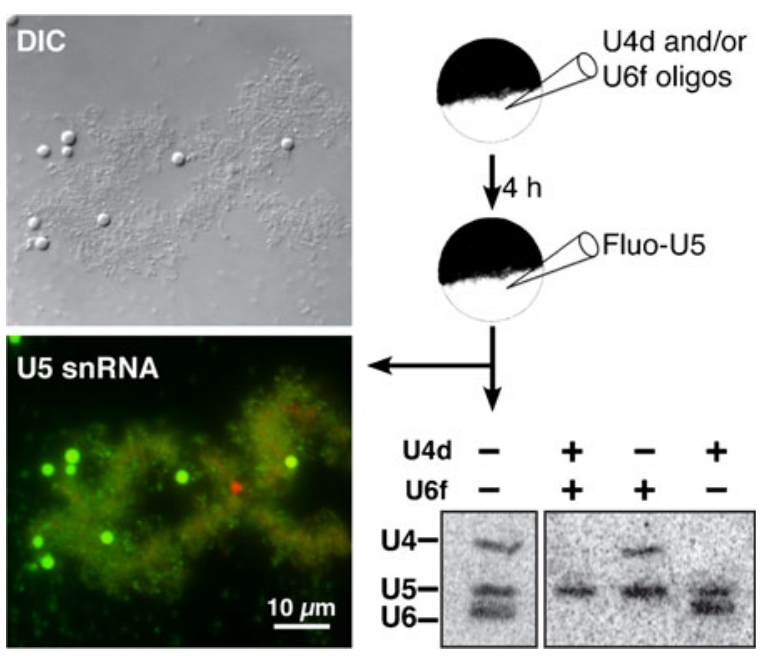

Fig. 2 U5 snRNPs can associate with nascent transcripts in the absence of U4 and U6 snRNAs. Fluorescently labeled U5 snRNAs were injected into the cytoplasm of oocytes depleted of U4 or/and U6 snRNAs, and nuclear spreads were prepared $18 \mathrm{~h}$ later. Newly formed U5 snRNPs associate with LBC loops and IGCs as strongly as when U4/U6 snRNAs are present in the oocyte. Northern blots show an efficient depletion of U4 and U6 snRNAs induced by oligonucleotide-targeted RNase H activity, while the level of U5 snRNA remains constant

(s) of the U5 snRNP acts to license its association with nascent transcripts, IGCs and CBs. To test this hypothesis, we performed a deletion analysis on the U5 snRNA. We used the predicted U5 snRNA secondary structure to guide our analysis, and the various mutants are presented in Table 1. Corresponding, fluorescein-labeled RNAs were synthesized and injected into the cytoplasm of stage IV-V Xenopus oocytes, and the subnuclear distributions of the newly formed snRNP complexes were analyzed by fluorescence microscopy on fixed nuclear spreads (Fig. 3). We found that none of the deletions downstream of the $\mathrm{Sm}$ binding site had an effect on the intranuclear trafficking of the newly assembled U5 snRNPs. In fact, Fig. 3 shows that U5 $3^{\prime} 96$ snRNP, which is missing stem-loop 2, still targeted CBs, IGCs, and LBC loops. In contrast, the deletion of the first 47 nucleotides completely inhibited the association of U5 $\Delta 5$ '47 snRNPs with IGCs and nascent transcripts. Interestingly, the removal of the first 47 nucleotides disrupts three predicted structures, stem-loop 1 (SL1), internal loop 1 (IL1), and internal loop 2 (IL2). These structures were then deleted individually (Table 1), and we found that while the removal of SL1 or IL2 had no effect, the deletion of IL1 was sufficient to abolish the interaction of U5 $\mathrm{IL} 1$ snRNPs with IGCs and LBCs (Fig. 3). Collectively, these data establish IL1 as the element of the U5 snRNP required for its association with nascent transcripts. In addition, as it is the case for U1 and U2 snRNPs, a deletion of most of the nucleotides upstream or downstream of the Sm site does not prevent U5 snRNPs from accumulating within CBs (Fig. 3; Table 1). It is likely, then, that the Sm site corresponds to the element responsible for targeting U5 snRNP to CBs.

Table 1 Localization of wild-type and mutant U5 snRNPs as defined on nuclear spreads

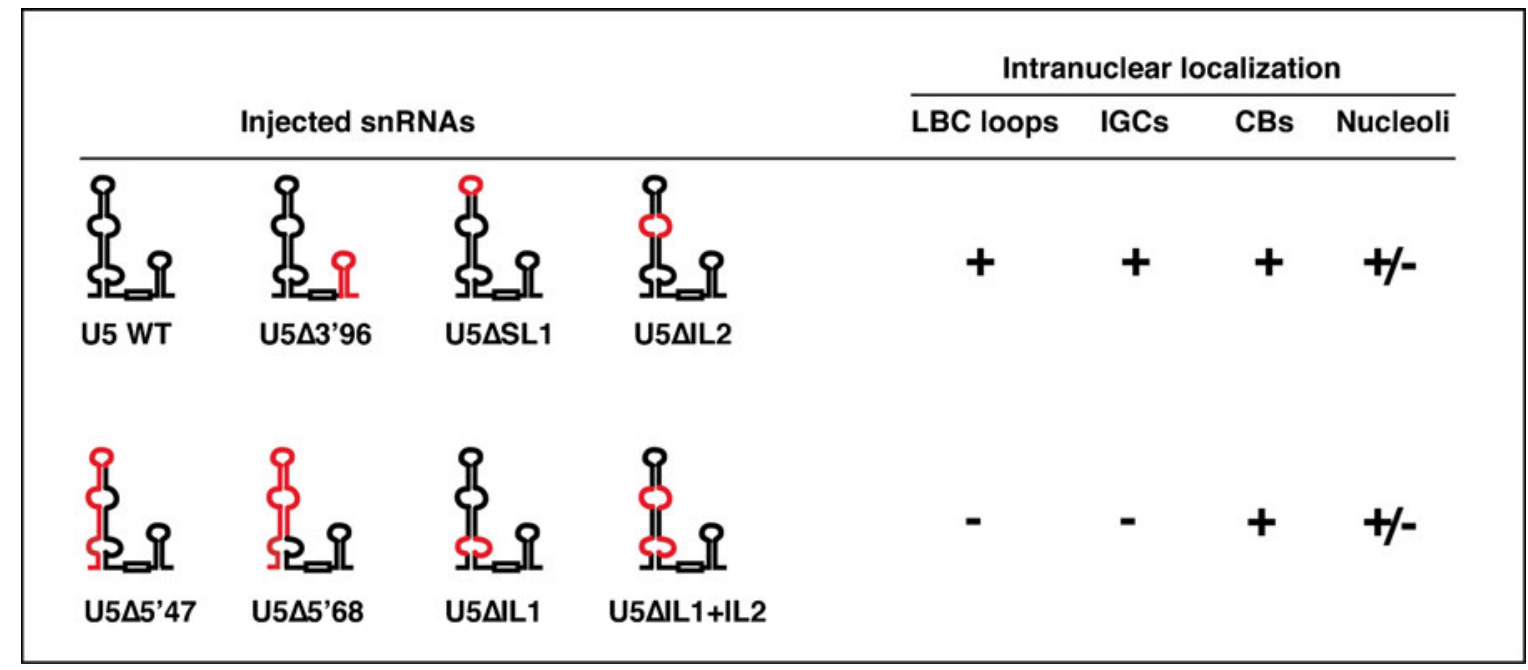


Fig. 3 The internal loop 1

(IL1) is critical for targeting U5 snRNP to nascent transcripts and IGCs. Fluorescently labeled, full-length U5 snRNA and various mutants were injected into stage IV-V oocytes. Nuclear spreads were prepared $18 \mathrm{~h}$ later, and the subnuclear distributions of newly formed U5 snRNPs were analyzed by immunofluorescence microscopy. Fluorescent micrographs show a merged image of the fluorescent RNAs (green) and the DNA (red). In the schematics of U5 snRNA mutants, deletions are shown as red lines
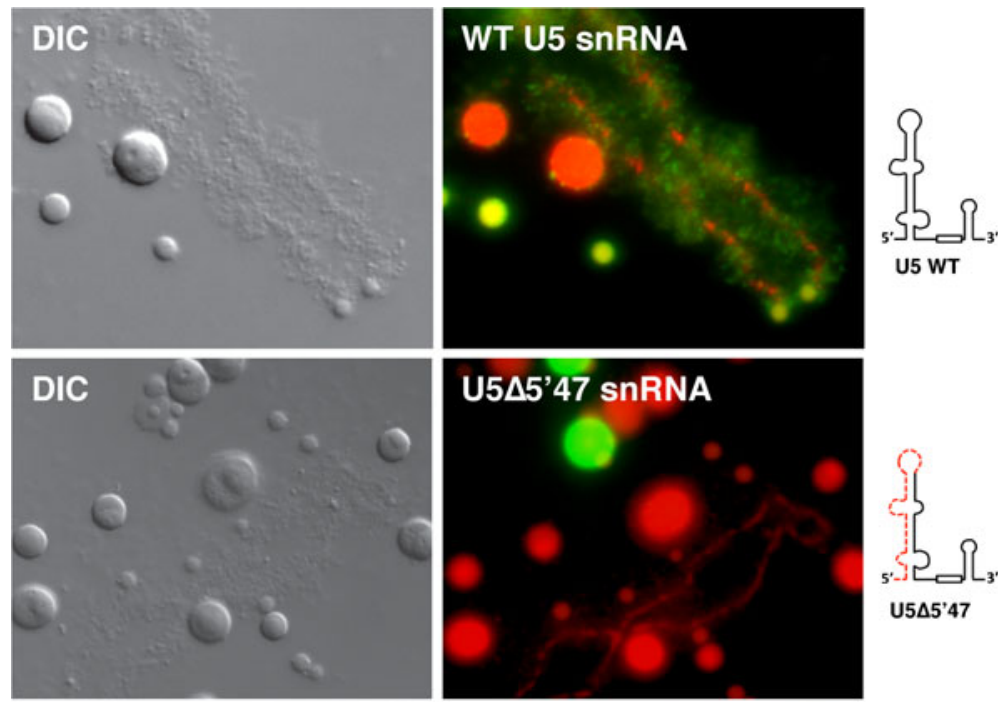
U5 $\triangle 5^{\prime} \mathbf{4} 7$
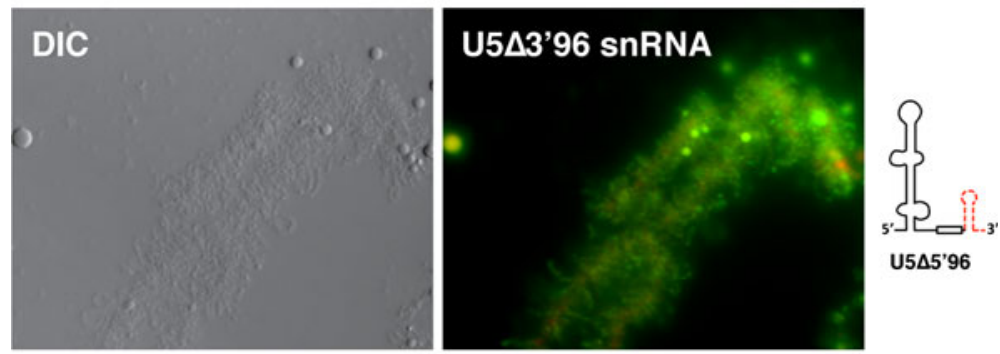

U5 $\triangle 5^{\prime} 96$
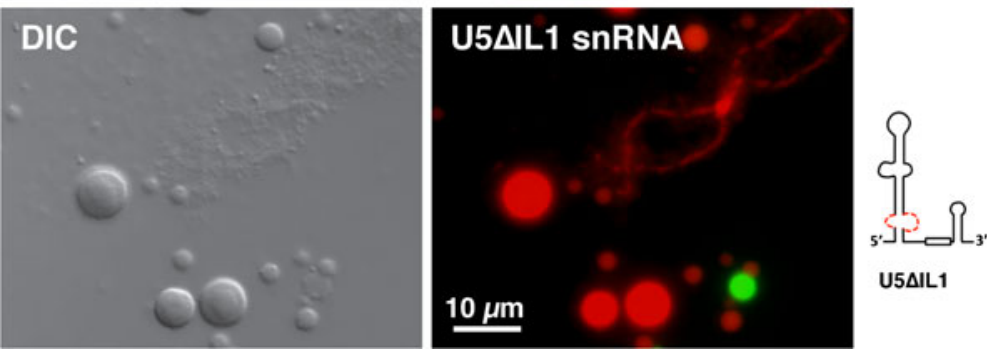

\section{Discussion}

While the biogenesis of the spliceosomal snRNPs is well documented (reviewed in Patel and Bellini 2008), the mechanisms that regulate their targeting to nascent transcripts still remain unclear. The current view is that snRNPs are recruited in a stepwise manner for the formation of the spliceosome on target pre-mRNAs. Recent findings, however, indicate that the recruitment of some snRNPs to nascent transcripts can occur in absence of spliceosomal assembly (Patel et al. 2007; Spiluttini et al. 2010; Paschedag et al., in revision). This conclusion stems from two main observations. First, the recruitment of snRNPs is unaffected by the absence of the U2 snRNP, which is critical for proper spliceosomal assembly and activity. Second, a non-functional snRNP that cannot comprise part of the spliceosome still associates with nascent transcripts. These data strongly suggest that distinct populations of snRNPs could co-exist on nascent transcripts. One possibility is that snRNPs are initially recruited to nascent transcripts, perhaps as a staging event to increase their local concentrations and allow efficient spliceosome formation upon the emergence during transcription of the necessary cis-acting RNA elements. Another possibility is that snRNPs may function in roles other than splicing. Interestingly, the U1 snRNP was recently shown to protect nascent transcripts from premature cleavage and polyadenylation 
independently of splicing (Kaida et al. 2010; Berg et al. 2012).

Importantly, then, these new insights emphasize the need to examine the regulatory processes by which the spliceosomal snRNPs are recruited to nascent transcripts in vivo. To our knowledge, LBCs are the only chromosomes where individual transcription units are readily visible by light microscopy, and we used them in this study to determine the elements of the U5 snRNP that are required for its association with RNAPII nascent transcripts. We previously demonstrated that the recruitment to nascent transcripts of U1 and U2 snRNPs is controlled by discrete elements of their respective RNA moieties: stem-loop 1 of U1 snRNA (Patel et al. 2007) and stem-loops 3 and 4 of U2 snRNA (Paschedag et al., in revision). Similarly, our deletion analysis of the U5 snRNP indicates that IL1 is required for its association with the LBC loops and IGCs. Since the U4/U6.U5 tri-snRNP is preassembled prior to integrating into the spliceosome, IL1 may act by promoting or stabilizing the association of the U5 snRNP with the U4/U6 di-snRNP. However, this possibility is directly ruled out by the fact that newly assembled U5 snRNPs still target nascent transcripts in oocytes depleted of the U4/U6 di-snRNP. In fact, we find that the intranuclear distribution of newly formed U5 snRNPs is unaffected by the depletion of the U4/U6 di-snRNP; this result agrees with a previous work showing that U5 targets nucleoli as a monosnRNP (Gerbi et al. 2003b). Thus, IL1 is likely to be a modular element of U5 snRNA that directly controls its intranuclear trafficking, presumably by acting through U5-associated proteins. Interestingly then, the U5 snRNP may also be the factor that targets the

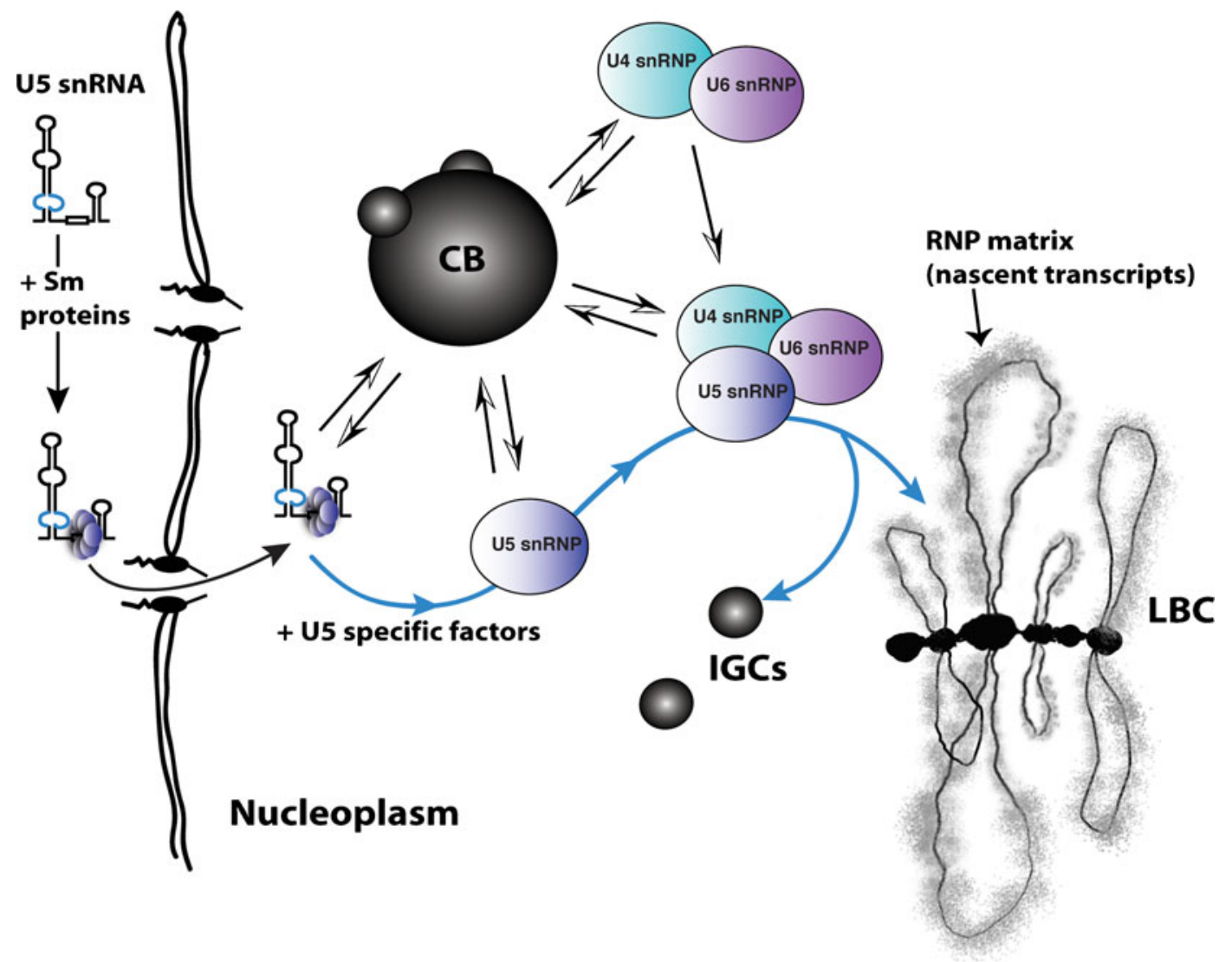

Fig. 4 Model of U5 snRNP intranuclear trafficking. After injection in the cytoplasm, fluorescent U5 snRNAs associate with a heteroheptameric ring of Sm proteins, which is sufficient upon nuclear entry for the association of newly formed U5 snRNPs with CBs. The interaction with CBs is dynamic, and further modification and assembly of U5 snRNPs and formation of the U4/U6.U5 tri-snRNP can occur either inside or outside of CBs. The IL1 structure (shown here in blue lines) of U5 snRNA, most likely bound by U5-specific proteins, is the element that eventually targets the U5 snRNP, and possibly the U4/U6.U5 tri-snRNP, to IGCs and nascent transcripts (LBC loops) 
U4/U6.U5 tri-snRNP to nascent transcripts and IGCs (as suggested in Fig. 4). Another interpretation is that the U4/U6.U5 tri-snRNP may assemble directly on transcripts after an initially recruitment of U5 snRNP and the U4/U6 di-snRNP but prior to spliceosomal assembly. We are currently testing whether the intranuclear trafficking of the U4/U6 di-snRNP is affected in oocytes lacking the U5 snRNP. Unfortunately, this work is being hindered by the fact that U5 snRNA could only be partially degraded using an antisense oligonucleotide strategy.

While IGCs and LBC loops are sites targeted by fully assembled snRNPs, CBs and nucleoli are often described as nuclear domains involved in the maturation and assembly of snRNPs (Gall et al. 1999; Yu et al. 2001; Gall 2003; Gerbi et al. 2003a; Matera 2003). In particular, CBs were directly implicated in the internal modification of the snRNAs by pseudouridylation and 2'-O-methylation (Darzacq et al. 2002; Jady et al. 2003). Here, we find that CBs accumulate newly formed wild-type and mutant U5 snRNPs at high concentrations. Interestingly, Gall et al. (1999) used in situ hybridizations on nuclear spread preparations more than a decade ago to reveal that endogenous U5 snRNPs, as well as all the other spliceosomal snRNPs, are present at a very low concentration in the oocyte CBs. We recently showed that the newly formed fluorescent U2 snRNPs that accumulate in CBs are incompletely assembled and lack the proteins that are required for targeting nascent transcripts (Paschedag et al., in revision). Similarly, the endogenous U1 snRNP rapidly accumulates in CBs upon truncation of the first stem-loop structure (U1SL1) of the U1 snRNA (Gall et al. 1999), which was subsequently shown to be both necessary and sufficient for targeting U1 snRNP to IGCs and LBC loops (Patel et al. 2007). It is likely, then, that the newly formed U5 snRNPs accumulated in CBs correspond primarily to incomplete RNP complexes. In addition, since the U5 snRNP comprises part of the tri-snRNP, a stoichiometric imbalance resulting from injecting fluorescent U5 snRNAs into oocytes may also contribute to the accumulation of fluorescent U5 snRNPs in CBs. Indeed, $\mathrm{CBs}$ are nuclear bodies where the formation of the U4/ U6.U5 tri-snRNP is enhanced (Stanek et al. 2003; Schaffert et al. 2004; Stanek and Neugebauer 2004), and one could speculate that monomeric U5 snRNP remains dynamically associated with $\mathrm{CBs}$ until assembly of the U4/U6.U5 tri-snRNP occurs.
We previously demonstrated that and U4 and U5 snRNPs target LBC loops in absence of spliceosomal assembly (Patel et al. 2007). Here, we present several pieces of data that further indicate a definite separation between the snRNP elements controlling intranuclear trafficking and those involved in catalyzing premRNA splicing. First, the deletion of SL1 has no effect on the intranuclear distribution of U5 snRNPs, at least qualitatively. In fact, U5 $\triangle \mathrm{SL} 1 \mathrm{snRNP}$ targets nascent transcripts and IGCs as efficiently as the wildtype U5 snRNP despite SL1 being essential for the splicing activity of the U5 snRNP, as shown by the deletion of only two nucleotides within the SL1 loop being sufficient to inhibit the second trans-esterification reaction of pre-mRNA splicing in vitro (O'Keefe and Newman 1998). Next, the splicing activity of U5 snRNAs lacking IL2 is severely hindered in vitro (Dix et al. 1998). Yet, we show here that the deletion of IL2 does not prevent the recruitment of U5 snRNPs to nascent transcripts. Finally, U5 snRNAs lacking IL1, which we show here as being unable to associate with nascent transcripts, are still able to catalyze splicing in vitro, albeit with a much lower efficiency that the wild-type U5 snRNA (Dix et al. 1998). Collectively, these data demonstrate a functional modularity of the U5 snRNP and an uncoupling of the elements that regulate its catalytic activity and its intranuclear trafficking.

Acknowledgments This work is supported by a grant from the National Science Foundation.

\section{References}

Austin C, Novikova N, Guacci V, Bellini M (2009) Lampbrush chromosomes enable study of cohesin dynamics. Chromosome Res 17:165-184

Bentley DL (2005) Rules of engagement: co-transcriptional recruitment of pre-mRNA processing factors. Curr Opin Cell Biol 17:251-256

Berg MG, Singh LN, Younis I et al (2012) U1 snRNP determines mRNA length and regulates isoform expression. Cell 150:53-64

Callan HG (1986) Lampbrush chromosomes. Mol Biol Biochem Biophys 36:1-252

Darzacq X, Jady BE, Verheggen C et al (2002) Cajal bodyspecific small nuclear RNAs: a novel class of 2'-O-methylation and pseudouridylation guide RNAs. EMBO J 21:2746-2756 
de Almeida SF, Carmo-Fonseca M (2008) The CTD role in cotranscriptional RNA processing and surveillance. FEBS Lett 582:1971-1976

Dix I, Russell CS, O'Keefe RT, Newman AJ, Beggs JD (1998) Protein-RNA interactions in the U5 snRNP of Saccharomyces cerevisiae. RNA 4:1675-1686

Gall JG (2003) A role for Cajal bodies in assembly of the nuclear transcription machinery. Tsitologiia 45:971-975

Gall JG, Bellini M, Wu Z, Murphy C (1999) Assembly of the nuclear transcription and processing machinery: Cajal bodies (coiled bodies) and transcriptosomes. Mol Biol Cell 10:4385-4402

Gerbi SA, Borovjagin AV, Lange TS (2003a) The nucleolus: a site of ribonucleoprotein maturation. Curr Opin Cell Biol 15:318-325

Gerbi SA, Borovjagin AV, Odreman FE, Lange TS (2003b) U4 snRNA nucleolar localization requires the NHPX/15.5-kD protein binding site but not Sm protein or U6 snRNA association. J Cell Biol 162:821-832

Jady BE, Darzacq X, Tucker KE et al (2003) Modification of Sm small nuclear RNAs occurs in the nucleoplasmic Cajal body following import from the cytoplasm. EMBO J 22:1878-1888

Jurica MS, Moore MJ (2003) Pre-mRNA splicing: awash in a sea of proteins. Mol Cell 12:5-14

Kaida D, Berg MG, Younis I et al (2010) U1 snRNP protects pre-mRNAs from premature cleavage and polyadenylation. Nature 468:664-668

Martins SB, Rino J, Carvalho T et al (2011) Spliceosome assembly is coupled to RNA polymerase II dynamics at the $3^{\prime}$ end of human genes. Nat Struct Mol Biol 18:1115-1123

Matera AG (2003) Cajal bodies. Curr Biol 13:R503

Morgan GT (2002) Lampbrush chromosomes and associated bodies: new insights into principles of nuclear structure and function. Chromosome Res 10:177-200
Nilsen TW (2003) The spliceosome: the most complex macromolecular machine in the cell? Bioessays 25:1147-1149

O'Keefe RT, Newman AJ (1998) Functional analysis of the U5 snRNA loop 1 in the second catalytic step of yeast premRNA splicing. EMBO J 17:565-574

Patel AA, Steitz JA (2003) Splicing double: insights from the second spliceosome. Nat Rev Mol Cell Biol 4:960-970

Patel SB, Bellini M (2008) The assembly of a spliceosomal small nuclear ribonucleoprotein particle. Nucleic Acids Res 36:6482-6493

Patel SB, Novikova N, Bellini M (2007) Splicing-independent recruitment of spliceosomal small nuclear RNPs to nascent RNA polymerase II transcripts. J Cell Biol 178:937-949

Schaffert N, Hossbach M, Heintzmann R, Achsel T, Luhrmann R (2004) RNAi knockdown of hPrp31 leads to an accumulation of U4/U6 di-snRNPs in Cajal bodies. EMBO J 23:3000-3009

Spiluttini B, Gu B, Belagal P et al (2010) Splicing-independent recruitment of U1 snRNP to a transcription unit in living cells. J Cell Sci 123:2085-2093

Stanek D, Neugebauer KM (2004) Detection of snRNP assembly intermediates in Cajal bodies by fluorescence resonance energy transfer. J Cell Biol 166:1015-1025

Stanek D, Rader SD, Klingauf M, Neugebauer KM (2003) Targeting of U4/U6 small nuclear RNP assembly factor SART3/p110 to Cajal bodies. J Cell Biol 160:505-516

Wallace RA, Jared DW, Dumont JN, Sega MW (1973) Protein incorporation by isolated amphibian oocytes. 3. Optimum incubation conditions. J Exp Zool 184:321-333

Yu YT, Shu MD, Narayanan A et al (2001) Internal modification of U2 small nuclear (sn)RNA occurs in nucleoli of Xenopus oocytes. J Cell Biol 152:1279-1288

Zhou Z, Licklider LJ, Gygi SP, Reed R (2002) Comprehensive proteomic analysis of the human spliceosome. Nature 419:182-185 